\title{
Year in Review 2018 by the Brazilian Gastrointestinal Tumors Group - GTG: Gastrointestinal Tumors
}

Revisão Ano 2018 pelo Grupo Brasileiro de Tumores Gastrointestinais - GTG: Tumores Gastrointestinais

Anelisa K. Coutinho ${ }^{1 *}$, Gabriel Prolla², Gustavo dos Santos Fernandes ${ }^{3}$, Heber Salvador ${ }^{4}$, Renata D’Alpino Peixoto ${ }^{5}$, Rui F. Weschenfelder 6 .

\begin{abstract}
In 2018, there were some important trials presented over multiple international meetings. The objective of this article is to summarize the most important and practice changing studies in gastrointestinal oncology this year. Some studies in gastroesophageal, pancreatic, colorectal and anal canal have been selected, including adjuvant therapy in pancreatic cancer, immunotherapy in metastatic gastroesophageal cancer, as well as strategies in regorafenib dose escalation and the role of hyperthermic intraperitoneal chemotherapy in metastatic colorectal.
\end{abstract}

Keywords: Gastrointestinal neoplasms; Stomach neoplasms; Esophageal neoplasms; Pancreatic neoplasms; Colorectal neoplasms; Anal canal.

\section{RESUMO}

Em 2018, houve alguns estudos importantes apresentados em múltiplas reuniões internacionais. O objetivo deste artigo é resumir os mais importantes estudos e mudança da prática oncológica gastrointestinal este ano. Alguns estudos gastroesofágicos, pancreáticos, colorretais e do canal anal foram selecionados, incluindo terapia adjuvante em câncer pancreático, imunoterapia em câncer gastroesofágico metastático, bem como estratégias de escalonamento da dose de regorafenibe e o papel da quimioterapia intraperitoneal hipertérmica em câncer colorretal metastático.

Descritores: Neoplasias gastrointestinais; Neoplasias estomacais; Neoplasias esofágicas; Neoplasias pancreáticas; Neoplasias colorretais; Canal anal.

1. Clinica AMO, Department of Oncology - Salvador - BA - Brazil

2. Escola de Medicina da PUCRS, Department of Oncology - Porto Alegre - RS - Brazil

3. Hospital Sírio Libanês, Department of Oncology - Brasília - DF - Brazil

4. AC Camargo Cancer Center, Department of Oncology - São Paulo - SP - Brazil

5. Hospital Alemão Oswaldo Cruz, Department of Oncology - São Paulo - SP - Brazil

6. Hospital Moinhos de Vento, Department of Oncology - Porto Alegre - RS - Brazil

Financial support: none to declare.

Conflicts of interest: The authors declare no conflict of interest relevant to this manuscript.

Correspondence author: Anelisa K. Coutinho. Clinica AMO, Department of Oncology

Email: coutinhoanelisa@uol.com.br

Received on: December 5, 2018 | Accepted on: June 27, 2019

DOI: $10.5935 / 1806-6054.20190014$ 


\section{INTRODUCTION}

Gastrointestinal (GI) tumors include at least 8 different entities of cancer located throughout the digestive tract and they represent a high proportion of all malignancies. Data regarding incidence and mortality in the U.S.A. estimate that colorectal cancer is the third malignancy in new cases and mortality in 2018. ${ }^{1}$ All together, the incidence of GI tumors is superior to the incidence of either breast or lung cancers. ${ }^{2}$ Besides, just considering the location, Gl tumors have a high morbidity potential, and consequently, every new promising treatment is welcome. The aim of this article is to present and review the most relevant studies presented or published during 2018 within a horizon of incorporation into clinical practice in a near future.

In September 2018, pembrolizumab was approved in Brazil for patients with metastatic PDL1 positive gastroesophageal cancer based on the results of the phase II open-label Keynote-059 trial. In this study, 259 patients (either PD-L1 positive or negative) received pembrolizumab 200 mg IV every 3 weeks. Objective response rate in the overall population was $11.6 \%$ (15.5\% for those with PD-L1 positive tumors) with a median duration of response of 8.4 months. Only $17.8 \%$ of the patients developed grade 3 or higher adverse events. ${ }^{3}$

\section{PANCREATIC CANCER}

The study PRODIGE24/CCTG.PA.6, presented at ASCO 2018, compared adjuvant mFOLFIRINOX versus gemcitabine in patients who had undergone complete macroscopic resection (R0 or R1) of a pancreatic adenocarcinoma. The study included 493 patients with ECOG PS of $0-1$, between the ages of 18 and 79 years and with a CA $19-9<180 \mathrm{U} / \mathrm{mL}$ within 12 weeks after surgery. The results demonstrated a significant improvement in disease-free survival (DFS) and overall survival (OS), as well as in metastasis-free survival and specific survival in all subgroup of patients. The median DFS improved from 12.8 months in the gemcitabine group to 21.6 months in the mFOLFIRINOX group of patients $(H R=0.58 ; 0.46-0.73 ; p<0.0001)$. The median OS improved from 35 months in the gemcitabine treated patients to 54.4 months in the mFOLFIRINOX treated patients ( $\mathrm{HR}=0.64 ; 0.48-0.86 ; \quad \mathrm{p}=0.003)$. Patients treated with mFOLFIRINOX had significantly more diarrhea, neutropenic fever, fatigue, vomiting, hand-foot syndrome, peripheral neuropathy and use of G-CSF while gemcitabine treated patients had more flu-like symptoms. However, toxicity was manageable and mFOLFIRINOX is the new standard in good performance status patients who have undergone complete resection of a pancreatic adenocarcinoma. ${ }^{4}$

\section{COLON CANCER}

ReDOS is a phase II study that evaluates a lower starting dose of regorafenib followed by a planned escalation compared to the standard dose in refractory metastatic colorectal cancer. The dose escalation started at $80 \mathrm{mg} /$ day in week one, with increasing dose in weeks 2 and 3 (120 mg/day and 160 $\mathrm{mg} /$ day, respectively) if no significant drug-related toxicities occurred. The standard dose arm started with the total dose of $160 \mathrm{mg} /$ day. Primary endpoint was the proportion of patients who completed 2 cycles of treatment with intention to initiate the third cycle, and was significantly higher among the experimental arm (43\% versus $25 \%, p=0.028$ ). This study brought a more acceptable schedule option into clinical practice and potentially establishes a new standard for optimizing regorafenib use. ${ }^{5}$

In 2017, the FDA approved anti-PD1 antibodies, such as pembrolizumab and nivolumab, for cancers demonstrated to have high microsatellite instability (MSI-H) or with mismatch repair deficiency (dMMR), regardless of tumor site or histology. ${ }^{6}$ This year during the ESMO meeting results of one cohort of the CheckMate 142, a nonrandomized phase II study, were presented. In this cohort, nivolumab plus low-dose ipilimumab were evaluated as firstline therapy in $45 \mathrm{MSI}-\mathrm{H} / \mathrm{dMMR}$ metastatic colorectal cancer patients. Results demonstrated a response rate of $60 \% 44.3-74.3$, disease control rate of $84 \% 70.5-93.5$ and a 12-months OS rate of 83\% 67.6-91.7. Median OS has not yet been reached. The combination was well tolerated, with only $16 \%$ of the patients experiencing grade 3-4 toxicities. Although early, these results indicate a potential new first line treatment option for patients with MSI-H/dMMR tumors. ${ }^{7}$

The phase III study PRODIGE 7 was presented at the ASCO meeting 2018 and demonstrated no benefit in adding HIPEC (hyperthermic intraperitoneal chemotherapy) to surgery in patients with colorectal peritoneal carcinomatosis. This multicenter trial randomized 265 patients with colorectal cancer and peritoneal metastasis for surgery only (non-HIPEC) versus surgery plus HIPEC. Results showed a median OS of 41.2 versus 41.7 months (HR=1.00 0.73-1.37, $p=0.995)$, respectively. Despite negative results, this was an expected and important trial, which raises questions regarding the benefit of HIPEC in colorectal cancer. ${ }^{8}$

The PROPHYLOCHIP trial aimed to define the role of a second-look surgery and HIPEC in patients at a high risk of developing colorectal peritoneal metastases. The high-risk group was defined as minimal peritoneal disease resected at the moment of the primary tumor surgery, a history of ovarian metastases, or perforated tumors. Between 2012 
and 2015, 150 patients were randomized to receive surveillance after adjuvant treatment or a second-look surgery, with cytoreduction plus HIPEC with oxaliplatin if any carcinomatosis were found. The selection criteria proved to be effective in detecting the high-risk group patients, since at the moment of the second-look procedure, $52 \%$ of them presented with peritoneal recurrence, with a median peritoneal cancer index of 4 and 25 patients (33\%) in the surveillance group experienced a peritoneal recurrence. Unfortunately, the primary end-point of the study was not met, with a 3 -year DFS of $44 \%$ in the second look group and $51 \%$ in the surveillance group $(p=0.75)$. The second end-point was also not met, with a 3-year OS of $80 \%$ and $79 \%$ in the surveillance and second-look group, respectively. The PROPHYLOCHIP study contributes to understanding the role of CRS in the management of peritoneal recurrence of colorectal cancer and confirmed the lack of benefit of the addition of HIPEC with oxaliplatin in this scenario. ${ }^{9}$

\section{RECTAL CANCER}

Long term results of the ADORE trial showed the benefit of adjuvant chemotherapy with oxaliplatin for patients with yp stage III rectal cancer. DFS was significantly improved for patients with yp stage III $(\mathrm{HR}=0.590 .38-0.92, \mathrm{p}=0.019)$, high grade histology $(H R=0.280 .08-0.97, p=0.045)$, tumors with minimal pathological regression $\quad(H R=0.40 \quad 0.19-0.85$, $\mathrm{p}=0.016)$, absence of lymphovascular $(\mathrm{HR}=0.55$ $0.34-0.88, p=0.013)$ or perineural invasion $(H R=1.53$ 0.33-0.86, $p=0.01)$. OS benefit was restricted to the group of patients with ypN2 (HR=0.42 0.18$0.96, p=0.04)$ and tumors with minimal pathological regression $(H R=0.420 .19-0.97, p=0.043) .{ }^{10}$

\section{ANAL CANAL}

A French multi-centric phase II trial enrolled 69 patients to evaluate clinical activity and safety of docetaxel, cisplatin, and fluorouracil (DCF) chemotherapy in good performance (ECOG 0-1) patients with metastatic or unresectable locally recurrent anal squamous cell carcinoma (ASCC). Sixty-six patients received treatment, 36 received the standard DCF (SDCF) regimen and 30 received the modified DCF (mDCF). Response rate (RR) was $86 \%$ with an impressive $44 \%$ CR rate. The median PFS for the overall population was 11.0 months (95\% Cl 9.3-16.4). OS at 12 months for the overall population was $83.1 \%(95 \% \mathrm{Cl} 71.5-90.3)$. No significant difference was found comparing the efficacy of mDCF to sDCF. As expected, toxicity was more frequent on the sDCF group with 30 of $3683 \%$ patients presenting at least one grade 3-4 adverse events and 16 of $3053 \%$ in the mDCF regimen. In our opinion, despite the single arm design, this clinical trial establishes mDCF as a first line option for ECOG 0-1 patients. ${ }^{11}$
Last, but not least, an international randomized phase II trial enrolled 91 patients with inoperable locally recurrent or metastatic treatment-naïve, advanced ASCC to receive cisplatin at $60 \mathrm{mg} / \mathrm{m}^{2}$ on D1 of a 21 day cycle plus 5-FU at $1000 \mathrm{mg} / \mathrm{m}^{2} /$ day over 24 hours on D1-D4, or carboplatin AUC 5 on D1 every 28 days plus paclitaxel at $80 \mathrm{mg} / \mathrm{m}^{2}$ on D1, 8 , and 15 every 28 days. The primary endpoint was RR, and secondary endpoints included PFS, OS, toxicity and quality of life (QoL). The RRs to treatment were $57.1 \%$ with cisplatin/5FU compared to $59.0 \%$ with carboplatin/paclitaxel. Median PFS was 5.7 months for cisplatin/5-FU versus 8.1 months for carboplatin/paclitaxel $(p=0.375)$ while median OS with the respective treatments were 12.3 and 20 months ( $H R=2.0 ; p=0.014)$. Cis/Fu was significantly more toxic and severe adverse events were reported in $62 \%$ of Cis/FU patients compared with $36 \%$ of patients receiving carboplatin/paclitaxel $(p=0.016)$. This trial in one of the few randomized trials on ACC and, despite the small sample size, the benefit in terms of OS should be taken into account. Therefore, we consider carboplatin/paclitaxel as a new standard of care for advanced/recurrent anal cancer. ${ }^{12}$

\section{REFERENCES}

1. Siegel RL, et al. Cancer Statistics, 2018. CA Cancer J Clin. 2018 jan;68(1):7-30.

2. Bray F, et al. Global Cancer Statistics 2018: GLOBOCAN estimates of incidence and mortality worldwide for 36 cancers in 185 countries. CA Cancer J Clin. 2018 nov;68(6):394-424.

3. Fuchs CS, et al. Safety and Efficacy of Pembrolizumab Monotherapy in Patients With
Previously Treated Advanced Gastric and Gastroesophageal Junction Cancer: Phase 2 Clinical KEYNOTE-059 Trial. JAMA Oncol. 2018 may;4(5):e180013.

4. Conroy T, et al. Unicancer GI PRODIGE24/CCTG PA.6 trial: A multicenter international randomized phase III trial of adjuvant mFOLFIRINOX versus gemcitabine (gem) in patients with resected pancreatic ductal adenocarcinomas. J Clin Oncol. 2018 jun;36(Suppl 18):LBA4001. 
5. Bekaii-Saab, et al. Regorafenib dose optimization study (ReDOS): Randomized phase II trial to evaluate escalating dosing strategy and preemptive topical steroids for regorafenib in refractory metastatic colorectal cancer (mCRC) - An ACCRU Network study. Ann Oncol. 2018jun;29(Suppl 5):0-014.

6. Lemery S, et al. First FDA approval agnostic of cancer site - when a biomarker defines the indication. N Engl J Med. 2017 oct;377(15):1409-1412.

7. Lenz $\mathrm{H}$-J, et al. Durable clinical benefit with Nivolumab plus low-dose Ipilimumab (IPI) as first-line therapy in microsatellite instability high/ mismatch repair deficient metastatic colorectal cancer. ESMO 2018 Congress; 2018 aug 22; Munich, Germany. ESMO. LBA18_PR

8. Quenet F, et al. A UNICANCER phase III trial of hyperthermic intraperitoneal chemotherapy (HIPEC) for colorectal peritoneal carcinomatosis (PC): PRODIGE 7. J Clin Oncol. 2018 jun;36(Suppl 18):LBA3503.

9. Goere D, et al. Results of a randomized phase 3 study evaluating the potential benefit of a second-look surgery plus HIPEC in patients at high risk of developing colorectal peritoneal metastases (PROPHYLOCHIP - NTC01226394). J Clin Oncol. 2018 jun;36(Suppl 15):3531.

10. Hong YS, et al. Long-term results of the ADORE trial: Adjuvant oxaliplatin, leucovorin, and 5-fluorouracil (FOLFOX) versus 5-fluorouracil and leucovorin (FL) after preoperative chemoradiotherapy and surgery for locally advanced rectal cancer. J Clin Oncol. 2018 jun;36(Suppl 15):3501.

11. Kim S, et al. Docetaxel, cisplatin, and fluorouracil chemotherapy for metastatic or unresectable locally recurrent anal squamous cell carcinoma (Epitopes-HPV02): a multicentre, single-arm, phase 2 study. Lancet Oncol. 2018 aug;19(8):1094-106.

12. Rao S, et al. InterAACT: A multicentre open label randomised phase II advanced anal cancer trial of cisplatin (CDDP) plus 5-fluorouracil (5- FU) vs carboplatin (C) plus weekly paclitaxel $(P)$ in patients (pts) with inoperable locally recurrent (ILR) or metastatic treatment naïve disease - An International Rare Cancers Initiative (IRCI) trial. ESMO 2018 Congress; 2018 aug 22; Munich, Germany. ESMO. LBA21. 